\title{
openheart Severe symptomatic aortic stenosis: medical therapy and transcatheter aortic valve implantation (TAVI) - a real-world retrospective cohort analysis of outcomes and cost-effectiveness using national data
}

\author{
Phillip M Freeman, ${ }^{1,2}$ Majd B Protty, ${ }^{1}$ Omar Aldalati, ${ }^{3}$ Arron Lacey, ${ }^{4}$ William King, ${ }^{1}$ \\ Richard A Anderson, ${ }^{5}$ Dave Smith ${ }^{3,4}$
}

To cite: Freeman PM, Protty MB, Aldalati 0 , et al. Severe symptomatic aortic stenosis: medical therapy and transcatheter aortic valve implantation (TAVI)-a realworld retrospective cohort analysis of outcomes and cost-effectiveness using national data. Open Heart 2016;3:e000414.

doi:10.1136/openhrt-2016000414

PMF and MBP have joint first authorship.

Received 20 January 2016 Revised 6 April 2016 Accepted 3 May 2016

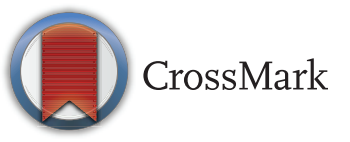

${ }^{1}$ Division of Population Medicine, Cardiff University, Cardiff, UK

2Department of Cardiology, Aalborg University Hospital, Aalborg, Denmark

${ }^{3}$ Morriston Hospital,

Swansea, UK

${ }^{4}$ Swansea University,

Swansea, UK

${ }^{5}$ University Hospital of Wales, Cardiff, UK

Correspondence to Dr Phillip M Freeman; phillip@fischerfreeman.com

\section{ABSTRACT}

Objectives: Determine the real-world difference between 2 groups of patients with severe aortic stenosis and similar baseline comorbidities: surgical turn down (STD) patients, who were managed medically prior to the availability of transcatheter aortic valve implantation (TAVI) following formal surgical outpatient assessment, and patients managed with a TAVI implant.

Design: Retrospective cohort study from real-world data.

Setting: Electronic patient letters were searched for patients with a diagnosis of severe aortic stenosis and a formal outpatient STD prior to the availability of TAVI (1999-2009). The second group comprised the first 90 cases of TAVI in South Wales (2009 onwards). 2 years prior to and 5 years following TAVI/STD were assessed. Patient data were pseudoanonymised, using the Secure Anonymized Information Linkage (SAIL) databank, and extracted from Office National Statistics (ONS), PatientEpisode Database for Wales (PEDW) and general practitioner databases.

Population: 90 patients who had undergone TAVI in

South Wales, and 65 STD patients who were medically managed.

Main outcome measures: Survival, hospital admission frequency and length of stay, primary care visits, and cost-effectiveness.

Results: TAVI patients were significantly older (81.8 vs $79.2)$, more likely to be male ( $59.1 \%$ vs $49.3 \%)$, baseline comorbidities were balanced. Mortality in TAVI versus STD was $28 \%$ vs $70 \%$ at 1000 days follow-up. There were significantly more hospital admissions per year in the TAVI group prior to TAVI/STD (1.5 (IQR 1.02.4) vs 1.0 IQR (0.5-1.5)). Post TAVI/STD, the TAVI group had significantly lower hospital admissions $(0.3$ (IQR 0.0-1.0) vs 1.2 (IQR $0.7-3.0)$ ) and lengths of stay (0.4 (IQR 0.0-13.8) vs 11.0 (IQR 2.5-28.5), p<0.05). The incremental cost-effectiveness ratio (ICER) for TAVI was $£ 10533$ per quality-adjusted life year (QALY).

\section{KEY QUESTIONS}

What is already known about this subject?

- Transcatheter aortic valve implantation (TAVI) is a transformational, minimally invasive technique that reduces symptoms, improves quality of life and decreases mortality for high-risk patients with severe symptomatic aortic stenosis. While mortality benefits are well proven, little realworld long-term data analysis of these groups is available in terms of survival, admission profiles and costs.

What does this study add?

- This study includes up to 10-year follow-up (maximum follow-up: 3812 days for medically managed surgical turn down patients, 1558 days for TAVI patients) of real-world patients and tracks all-cause mortality as well as long-term readmission details using linked anonymised data. For the first time, we see the direct cost of both these management strategies together with a cost-effectiveness analysis.

How might this impact on clinical practice?

- While this is a relatively small study, it represents an important step in real-world long-term outcomes of either medical therapy or TAVI in a high-risk population with severe symptomatic aortic stenosis. It also supports clinical trial findings of improved outcomes in patients managed with TAVI and for the first time reveals that TAVI is a cost-effective procedure in realworld practice.

Conclusions: TAVI patients were more likely to survive and avoid hospital admissions compared with the medically managed STD group. The ICER for TAVI was $£ 10533$ per QALY, making it a cost-effective procedure. 


\section{INTRODUCTION}

Aortic stenosis is the most common valvular heart disease which affects $4.6 \%$ of adults older than 75 years, and is associated with significant morbidity and mortality. ${ }^{1}$ Given the association between age and heart valve disease, numbers of patients with severe aortic stenosis are predicted to more than double by 2050 in Europe and the USA. ${ }^{2}$ Historically, patients who were deemed to be at high risk of surgical complication were treated using medical therapy.

Transcatheter aortic valve implantation (TAVI) is a transformational, minimally invasive technique that reduces symptoms, improves quality of life and decreases mortality for high-risk patients with severe symptomatic aortic stenosis. ${ }^{3}$ While mortality benefits are well proven, ${ }^{4-7}$ little real-world long-term data analysis of these groups is available in terms of survival, admission profiles and costs. Brecker $e t a l^{8}$ published an analysis of cost-effectiveness of TAVI versus medical management (STD) that used a number of data sources (Corevalve ADVANCE study ${ }^{9}$ and Partner $B^{4}$ ). This study suggested that TAVI was a highly cost-effective therapy but noted the limitations of modelling outcomes and costs with these data in respect of the US context (for cost analysis). ${ }^{10}$ We aimed to extend the Brecker $e t$ t $a$ s study using real-world data and outcomes from a single national data set that was able to capture all readmissions, local costs and outcomes.

In this study, we set out to address these gaps in the literature by examining routinely collected real-world national data in Wales to determine the impact of TAVI on mortality, hospital admissions and cost-effectiveness in real-life practice.

\section{METHODS}

\section{Data sources}

We conducted a retrospective cohort analysis using aortic stenosis registries from the two tertiary cardiac centres in Wales, linked with secondary care data from the Patient Episode Database for Wales (PEDW), primary care data from general practices in Wales and mortality data from the Office of National Statistics (ONS).

\section{Aortic stenosis registry}

Electronic patient letters from University Hospital of Wales (Cardiff, UK) and Morriston Hospital (Swansea, UK) were searched for patients with a diagnosis of severe aortic stenosis and a formal outpatient surgical turn down (STD) prior to the availability of TAVI (19992009) to generate the medically managed cohort of patients. The second group was the first 90 cases of TAVI in South Wales (2009 onwards) from both hospitals. The type of TAVI that was used by University Hospital of Wales was self-expanding valves (CoreValve) whereas Morriston Hospital used balloon-expanding valves (Edwards).

\section{Primary and secondary care data}

Data for both cohorts were collected from the primary care and secondary care database to cover a period of 2 years prior and 5 years following TAVI or STD. These data included dates and durations of all hospital admissions as well as all visits to see a general practitioner (GP).

\section{Mortality data}

These were extracted from the ONS to include date and cause of death as documented on the official death certificate.

\section{Data linkage}

These sources of data were pseudoanonymised and linked using the Secure Anonymized Information Linkage (SAIL) databank. This databank is a national Welsh database that links the widest possible range of person-based data using robust anonymisation techniques. ${ }^{11}{ }^{12}$ It contains over 500 million person-based records and is operated and administered by the Health Information Research Unit (HIRU), Swansea University, for the purposes of health-related real-world research.

\section{Outcomes}

Our primary outcomes were to determine the difference in rates of mortality, admission profiles and associated healthcare costs in a real-world setting for two groups of patients with severe symptomatic aortic stenosis: STD patients, who were managed medically prior to the availability of TAVI, and patients managed with a TAVI implant.

\section{Statistical analysis}

Analysis was carried out using SPSS V.19.0 statistics package (IBM, New York, USA) and Microsoft Excel (Microsoft, California, USA). The Charlson comorbidity index was calculated directly from International Classification of Diseases (ICD)-10 diagnoses within the database using Structure Query Language (SQL) code, relying on previously described comorbidity scoring systems as modified by Bottle and Aylin. ${ }^{13} 14$

Continuous variables with normal distribution are presented in tabular form as mean $\pm \mathrm{SD}$, whereas nonnormally distributed variables are presented as median with IQR. Patient characteristics were described as frequencies and percentages with $\chi^{2}$ analysis; mortality was examined using Kaplan-Meier survival analysis and log-rank testing; and admission profile data, including Charlson scores, was compared using Student's t-test for parametric data or Mann-Whitney $U$ test for non-parametric data. Significance levels were taken at a $\mathrm{p}$ value $<0.05$.

\section{Economic model}

In order to report the economic results of this analysis as transparently as possible, a simple decision analytic model was used to assess the long-term cost-effectiveness of TAVI compared with no treatment. As patient 
mortality data were directly available from the centres own activity, the primary objective of the model was to account the potential costs and quality of life outcomes and quantify the projected values over time.

The computer simulation used was a deterministic decision analytic tool incorporating a cost-utility element to evaluate two treatment arms, that is, TAVI and no intervention. Transition probabilities were derived from the Kaplan-Meier curves for each arm and used to account mortality at 30 days, 6 months, 1 year and annually subsequent to that. The chosen time horizon was 5 years, which is in line with the follow-up data available for the TAVI arm. The model accounted costs from the payer perspective (the National Health Service) and these were limited to the cost of the TAVI intervention (£22 000 to reflect a typically reimbursed UK hospital payment) and annual costs of readmissions derived from the original study. For the untreated arm, these were inflated to 2012 values using the hospital and community health services index. Quality-adjusted life expectancy measured in quality-adjusted life-years (QALYs) was quantified using the published utility values from the partner B study. ${ }^{4}$ It was assumed that there were no changes in quality of life after the first year.

All clinical and economic outcomes were discounted annually at $3.5 \%$ in line with UK guidelines. Discounting is a standard method applied to the projection of future outcomes that accounts for the biased value of benefits that occur now versus the future.

\section{RESULTS}

There were a total of 90 patients in our data set who had undergone TAVI and 65 patients who were medically

\begin{tabular}{|c|c|c|c|}
\hline ICD diagnosis & STD $(n=65)$ & TAVI $(n=90)$ & $\begin{array}{l}p \\
\text { Value }\end{array}$ \\
\hline Age & $79.2( \pm 6.59)$ & $81.8( \pm 7.91)$ & $<0.05$ \\
\hline Sex (female) & $50.70 \%$ & $40.90 \%$ & $<0.01$ \\
\hline Anaemia & $11(20.37 \%)$ & 29 (36.25\%) & 0.035 \\
\hline Cancer & $7(12.96 \%)$ & 17 (21.25\%) & 0.157 \\
\hline Stroke & $4(7.41 \%)$ & $11(13.75 \%)$ & 0.168 \\
\hline COPD & $9(16.67 \%)$ & 21 (26.25\%) & 0.144 \\
\hline Renal failure & $11(20.37 \%)$ & $9(11.25 \%)$ & 0.105 \\
\hline Diabetes & 20 (37.04\%) & $32(40.00 \%)$ & 0.736 \\
\hline LVF & 32 (59.26\%) & $54(67.50 \%)$ & 0.464 \\
\hline $\begin{array}{l}\text { Pulmonary } \\
\text { emboli }\end{array}$ & $2(3.70 \%)$ & $2(2.50 \%)$ & 0.629 \\
\hline $\begin{array}{l}\text { Pulmonary } \\
\text { hypertension }\end{array}$ & $1(1.85 \%)$ & $0(0.00 \%)$ & 0.174 \\
\hline PVD & $5(9.26 \%)$ & $12(15.00 \%)$ & 0.244 \\
\hline Recent MI & $17(31.48 \%)$ & 32 (40.00\%) & 0.314 \\
\hline Charlson index & $10.63( \pm 9.95)$ & $15.81( \pm 10.46)$ & $<0.01$ \\
\hline
\end{tabular}

managed after STD. Maximum (mean) follow-up duration was 1558 (438) days for TAVI patients and 3812 (728) days for STD patients. Table 1 compares the baseline characteristics for the two groups. Patients in the TAVI group were more likely to be older, male, to have a previous diagnosis of anaemia and to have a higher Charlson comorbidity index. Other baseline characteristics were broadly similar between the two groups.

Survival analysis shows that patients who were managed with TAVI were more likely to survive compared with the STD group $(\mathrm{p}<0.05)$ at 3 years follow-up (figure 1). Survival rates at 1000 days follow-up were $72 \%$ for the TAVI group and $30 \%$ for the STD group.

A comparison of the number of hospital admissions per year for both groups before and after the date of the TAVI procedure (TAVI group) or STD (STD group) can be seen in figure 2. TAVI patients had significantly fewer hospital admissions per year after their procedure compared with the STD group (0.3 (IQR 0.0-1.0) vs 1.2 (IQR 0.7-3.0), $\mathrm{p}<0.05$ ), despite the reverse being true before their procedure (1.5 (IQR 1.0-2.4) vs 1.0 IQR $(0.5-1.5), \mathrm{p}<0.05)$.

Following the procedure/turn-down date, the length of hospital stay was significantly less in the TAVI group compared with the STD group (0.4 (IQR 0.0-13.8) vs 11.0 (IQR 2.5-28.5), $\mathrm{p}<0.05$ ), as shown in figure 3. There was no significant difference in the number of visits to the GP for either group before and after the procedure/turn-down date (figure 4).

A cost-effectiveness analysis can be seen in table 2 and figure 5. This demonstrates that the incremental

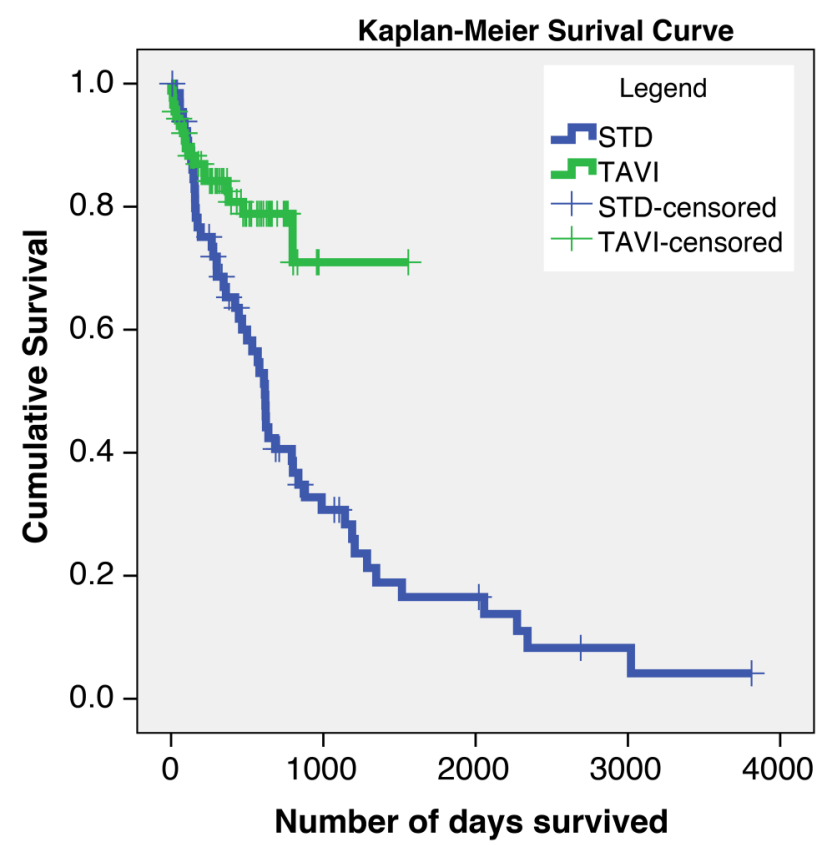

Figure 1 Kaplan-Meier survival curves comparing the STD cohort (blue) and TAVI cohort (green). Patients who are lost to follow-up are censored and are shown as crosses on each curve $(p<0.05)$. STD, surgical turn down; TAVI, transcatheter aortic valve implantation. 


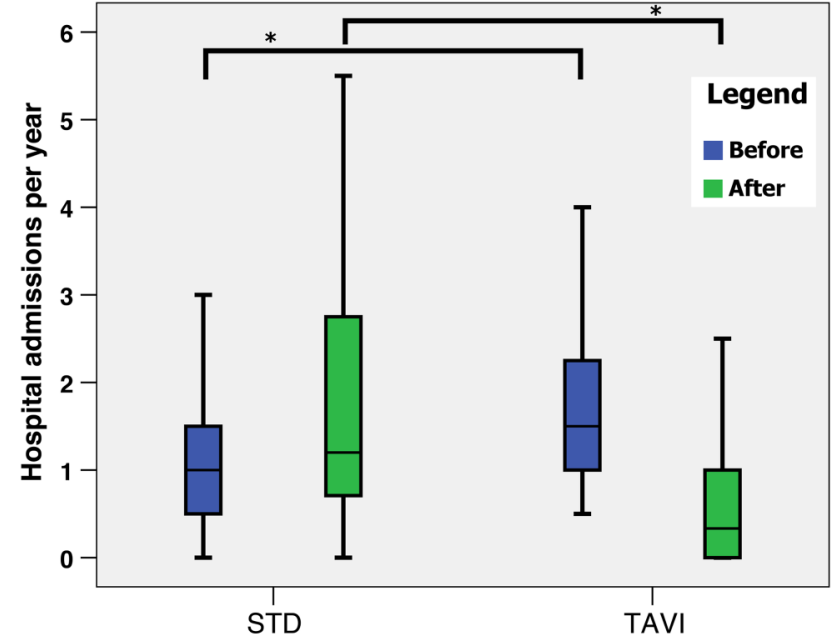

Figure 2 Box plot of frequency of hospital admissions for the STD and TAVI groups before (blue) and after (green) the $\mathrm{TAVI} /$ turn-down date. Boxes indicate the IQRs with a central band corresponding to the median, whereas the 'whiskers' indicate upper and lower limits. ${ }^{*} p<0.05$. Mann-Whitney $U$ test for non-parametric data. NS, not significant; STD, surgical turn down; TAVI, transcatheter aortic valve implantation.

cost-effectiveness ratio is $£ 10533$ per QALY gained from a TAVI procedure.

\section{DISCUSSION}

\section{Main findings}

This study reveals that patients with severe aortic stenosis who went on to have TAVI treatment had lower mortality rates, as well as less frequent and shorter subsequent hospital admissions than their medically managed

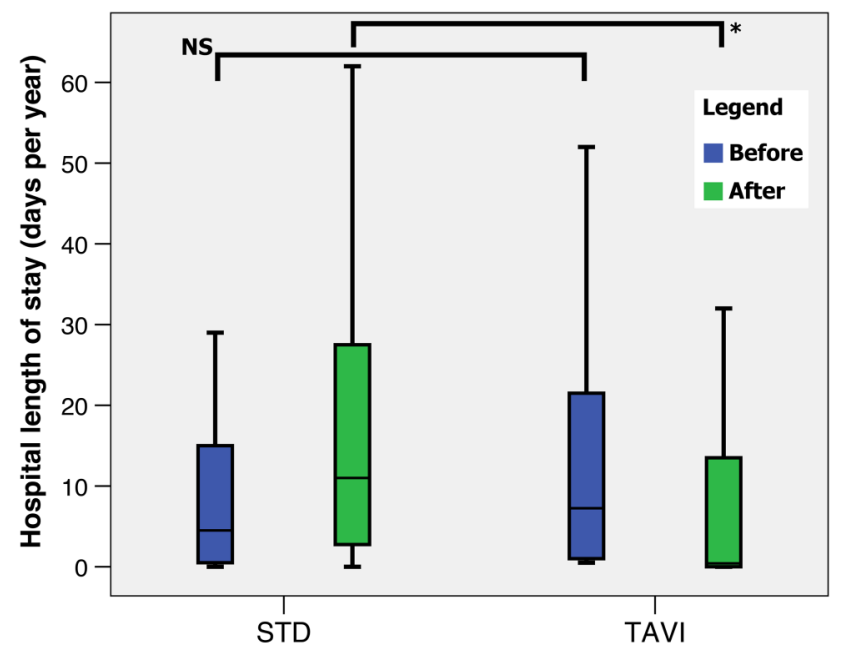

Figure 3 Box plot of hospital length of stay (in days) for the STD and TAVI groups before (blue) and after (green) the TAVI/turn-down date. Boxes indicate the IQRs with a central band corresponding to the median, whereas the 'whiskers' indicate upper and lower limits. ${ }^{*} p<0.05$. Mann-Whitney $U$ test for non-parametric data. NS, not significant; STD, surgical turn down; TAVI, transcatheter aortic valve implantation.

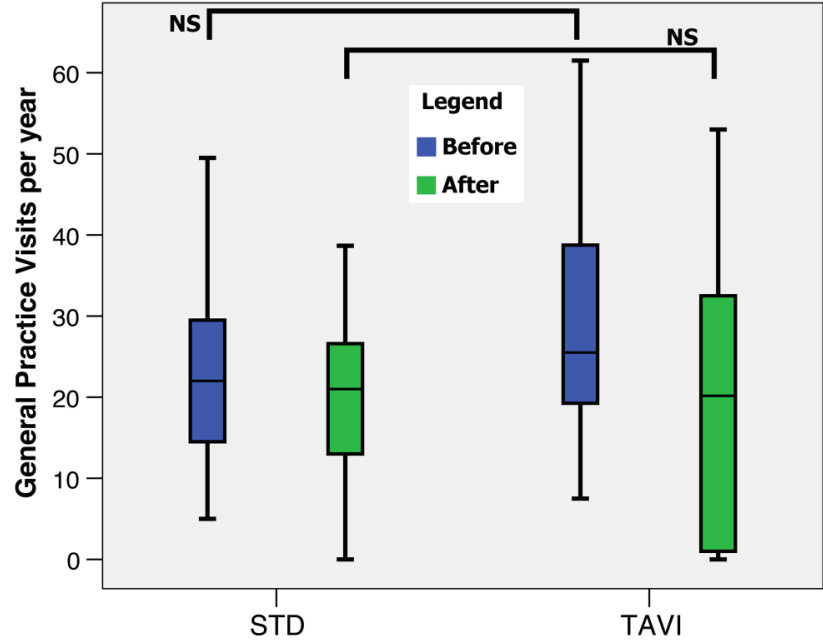

Figure 4 Box plot of frequency of general practice visits for the STD and TAVI groups before (blue) and after (green) the TAVI/turn-down date. Boxes indicate the IQRs with a central band corresponding to the median, whereas the 'whiskers' indicate upper and lower limits. Mann-Whitney $U$ test for non-parametric data. NS, not significant; STD, surgical turn down; TAVI, transcatheter aortic valve implantation.

counterparts (STD group). Our data again confirm the stark prognosis of untreated aortic stenosis with only $30 \%$ of patients alive at 1000 days of follow-up. Moreover, the incremental cost-effectiveness ratio of $£ 10533$ per QALY gained from undergoing TAVI gives this procedure a favourable cost-benefit balance.

\section{Strengths and limitations}

The most important strength of this study is the use of real-life individual patient data with linkage of local and national databases in Wales. This reduces selection bias, which may accompany carefully selected clinical trial groups. Additionally, this is the first study that examines the real-life cost-effectiveness of undergoing TAVI using multiple outcome measures.

Selection of medical cohort (STD following formal outpatient referral to a consultant cardiothoracic surgeon) helped identify a cohort that was similar to the contemporary TAVI population. This has been confirmed by the exploring baseline characteristics. In fact, on comparing the baseline comorbidities using the

Table 2 QALYs and ICER in TAVI and STD patients

\begin{tabular}{llll}
\hline & TAVI & STD & Difference \\
\hline Life-years & 3.82 & 2.09 & 1.73 \\
QALYs & 2.47 & 1.18 & 1.29 \\
Costs & $£ 44751$ & $£ 31096$ & $£ 13655$ \\
ICER & $£ 10533 / Q A L Y$ & gained & \\
\hline ICER, incremental cost-effectiveness ratio; QALY, quality-adjusted \\
life-year; STD, surgical turn down; TAVI, transcatheter aortic valve \\
implantation.
\end{tabular}




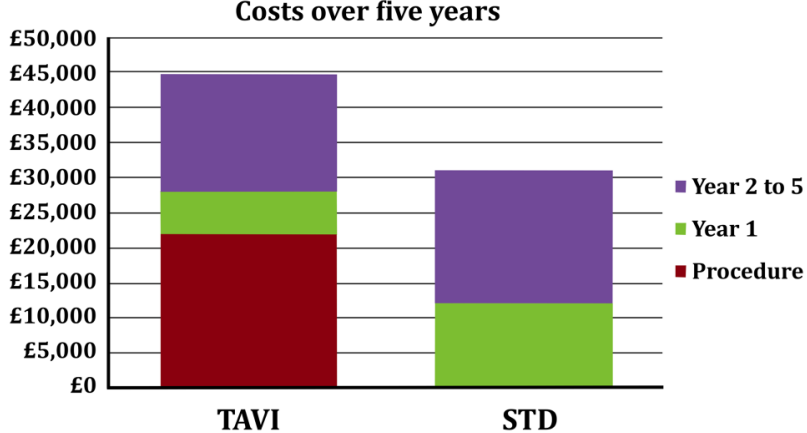

Figure 5 Incremental bar chart for the costs incurred in the TAVI and STD populations. Red corresponds to the cost of procedure (if any), green corresponds to the costs in the first year after the index date, whereas purple corresponds to costs in years $2-5$.

Charlson index, the TAVI cohort was significantly more likely to have a higher comorbidity score.

The main limitation to this study is common with other studies that use retrospective routinely collected data, which may not have been designed for the purposes of research and audit studies. These limitations include record keeping issues, which may result in the potential 'loss' of some critical data, due to administrative or data inputting issues. When comparing the baseline characteristics of groups, there may be hidden characteristics that are not evident in our data; however, we have used as many characteristics as possible including a full analysis of comorbidity using the Charlson index variables. Other limitations that need to be considered include interoperator and interhospital variability of outcomes and the difference in the type of valve used by each of the hospitals, with University Hospital of Wales using self-expanding valves (CoreValve) and Morriston Hospital using balloon-expanding valves (Edwards).

\section{Economic modelling}

In contrast to other published economic models of TAVI, there was no distinction between short-term (30 days) and subsequent clinical events. The details of perioperative complications such as stroke and bleeding events were not accounted. Although this may to some degree alter the actual patient quality of life benefit due to the difference of TAVI implant performance, the impact on mortality is fully captured, as are the costs from the payer perspective.

It is worth noting that the economic model and cost accounting does not capture primary care costs (GP visits). It was assumed that these were negligible compared with the costs of rehospitalisations. In addition, it was assumed that the annual costs of rehospitalisation did not increase over time. This may be an underestimation of the total costs but given the time horizon selected and the discounting applied, it was thought that it would not affect the overall conclusions.

\section{Comparison with other studies}

The landmark clinical trial that has demonstrated a mortality benefit with TAVI over medical management in inoperable patients was the Placement of Aortic Transcatheter Valves (PARTNER) multicentre trial (cohort B) which included 358 patients. ${ }^{4-7}$ The 3-year survival in that trial was $45.9 \%$ in the TAVI (Edwards balloon-expandable) group and $19.1 \%$ in the medically managed group. Given the mortality benefit that was demonstrated in the PARTNER trial, it was not possible to perform clinical trials to directly compare the effects of self-expanding TAVI (CoreValve) with medically managed patients, although a prospective single arm trial using historical controls for comparison demonstrated a mortality benefit with this type of valve. ${ }^{15}$

Given the above, and the corroborating data from our study showing that survival rates in aortic stenosis are better with TAVI, it should be noted that our real-life survival rates were markedly better for both groups at $72 \%$ for TAVI and $30 \%$ for the medically managed (STD) group. Additionally, the 3-year mortality rate for TAVI-treated patients published by the UK TAVI registry studies was higher $(38.8 \%)$ than that shown in our study $(28 \%) .{ }^{16}$

There are no fully published studies that examine the cost-effectiveness of TAVI compared with medical management of inoperable patients with severe aortic stenosis. However, a recent model-based economic evaluation demonstrated that although TAVI is more costly than medical management, it is more effective, and as a result, it was associated with an ICER of $£ 12900$ per QALY, deeming it cost-effective. ${ }^{17}$ Our current study demonstrates a real-life ICER of $£ 10533$ per QALY, a more cost-effective figure than previous economic evaluations.

\section{Implications for practice and future research}

Despite the limited numbers in this study, there is a marked improvement in mortality and morbidity rates that can be seen in patients who undergo TAVI compared with medical management only. Longer follow-up studies will be interesting to assess long-term outcomes, which can be derived from routinely collected national data as was demonstrated by our study.

In conclusion, our results support the use of TAVI where possible as a cost-effective and clinically effective intervention in patients with severe aortic stenosis who are deemed to be at high risk for conventional surgical valve replacement.

Contributors All authors contributed to the acquisition of the data. MBP, AL, WK and PMF contributed to linking the data to national databanks. MBP, $O A$, WK, RAA, DS and PMF contributed to the study design. MBP, AL, PMF and DS contributed to the analysis of the data. MBP and PMF drafted the manuscript. All authors contributed to the interpretation of the data and the revision of the work, and all approved the final version to be published. The authors of this report are responsible for its content, and affirm that the manuscript is an honest, accurate and transparent account of the study being reported; that no important aspects of the study have been omitted; and that any discrepancies from the study as planned have been explained.

Competing interests None declared. 
Provenance and peer review Not commissioned; externally peer reviewed.

Data sharing statement No additional data are available.

Open Access This is an Open Access article distributed in accordance with the Creative Commons Attribution Non Commercial (CC BY-NC 4.0) license, which permits others to distribute, remix, adapt, build upon this work noncommercially, and license their derivative works on different terms, provided the original work is properly cited and the use is non-commercial. See: http:// creativecommons.org/licenses/by-nc/4.0/

\section{REFERENCES}

1. Nkomo VT, Gardin JM, Skelton TN, et al. Burden of valvular heart diseases: a population-based study. Lancet 2006;368:1005-11.

2. Osnabrugge RL, Mylotte D, Head SJ, et al. Aortic stenosis in the elderly: disease prevalence and number of candidates for transcatheter aortic valve replacement: a meta-analysis and modeling study. J Am Coll Cardiol 2013;62:1002-12.

3. Gooley R, Cameron JD, Meredith IT. Transcatheter aortic valve implantation-yesterday, today and tomorrow. Heart Lung Circ 2015;24:1149-61.

4. Leon MB, Smith CR, Mack M, et al. Transcatheter aortic-valve implantation for aortic stenosis in patients who cannot undergo surgery. N Engl J Med 2010;363:1597-607.

5. Makkar RR, Fontana GP, Jilaihawi $\mathrm{H}$, et al. Transcatheter aortic-valve replacement for inoperable severe aortic stenosis. N Engl J Med 2012;366:1696-704.

6. Kapadia SR, Tuzcu EM, Makkar RR, et al. Response to letter regarding article, "long-term outcomes of inoperable patients with aortic stenosis randomly assigned to transcatheter aortic valve replacement or standard therapy". Circulation 2015;132:e118-19.

7. Kapadia SR, Tuzcu EM, Makkar RR, et al. Long-term outcomes of inoperable patients with aortic stenosis randomly assigned to transcatheter aortic valve replacement or standard therapy. Circulation 2014;130:1483-92.
8. Brecker S, Mealing S, Padhiar A, et al. Cost-utility of transcatheter aortic valve implantation for inoperable patients with severe aortic stenosis treated by medical management: a UK cost-utility analysis based on patient-level data from the ADVANCE study. Open Heart 2014;1:e000155.

9. Linke A, Wenaweser P, Gerckens U, et al. Treatment of aortic stenosis with a self-expanding transcatheter valve: the International Multi-centre ADVANCE Study. Eur Heart J 2014;35:2672-84.

10. Reynolds MR, Cohen DJ. The cost-effectiveness of transcatheter aortic valve replacement for nonsurgical candidates: revisionist history or the final word? Circ Cardiovasc Qual Outcomes 2013;6:376-8.

11. Ford DV, Jones KH, Verplancke JP, et al. The SAIL Databank: building a national architecture for e-health research and evaluation. BMC Health Serv Res 2009;9:157.

12. Lyons RA, Jones $\mathrm{KH}$, John $\mathrm{G}$, et al. The SAIL databank: linking multiple health and social care datasets. BMC Med Inform Decis Mak 2009;9:3.

13. Charlson ME, Pompei P, Ales KL, et al. A new method of classifying prognostic comorbidity in longitudinal studies: development and validation. J Chronic Dis 1987;40:373-83.

14. Bottle A, Aylin P. Comorbidity scores for administrative data benefited from adaptation to local coding and diagnostic practices. J Clin Epidemiol 2011;64:1426-33.

15. Popma JJ, Adams DH, Reardon MJ, et al. Transcatheter aortic valve replacement using a self-expanding bioprosthesis in patients with severe aortic stenosis at extreme risk for surgery. J Am Coll Cardiol 2014;63:1972-81.

16. Duncan A, Ludman $P$, Banya $W$, et al. Long-term outcomes after transcatheter aortic valve replacement in high-risk patients with severe aortic stenosis: the U.K. Transcatheter Aortic Valve Implantation Registry. JACC Cardiovasc Interv 2015;8:645-53.

17. Orlando R, Pennant M, Rooney S, et al. Cost-effectiveness of transcatheter aortic valve implantation (TAVI) for aortic stenosis in patients who are high risk or contraindicated for surgery: a model-based economic evaluation. Health Technol Assess 2013;17:1-86. 